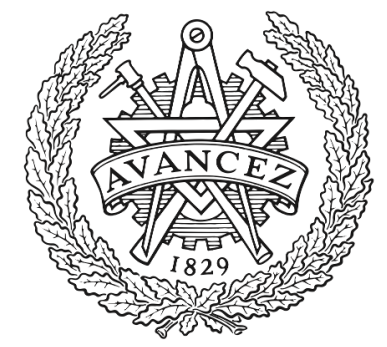

CHALMERS

UNIVERSITY OF TECHNOLOGY

\title{
Low-loss high-Q silicon-rich silicon nitride microresonators for Kerr nonlinear optics
}

Downloaded from: https://research.chalmers.se, 2023-04-26 15:33 UTC

Citation for the original published paper (version of record):

Ye, Z., Fülöp, A., Helgason, Ò. et al (2019). Low-loss high-Q silicon-rich silicon nitride microresonators for Kerr nonlinear optics. Optics Letters, 44(13): 3326-3329.

http://dx.doi.org/10.1364/OL.44.003326

N.B. When citing this work, cite the original published paper. 


\title{
Low-loss high-Q silicon-rich silicon nitride microresonators for Kerr nonlinear optics
}

\author{
Zhichao Ye, ${ }^{1,}{ }^{*}$ Attila Fülöp, ${ }^{1}$ Óskar Bjarki Helgason, ${ }^{1}$ Peter A. \\ ANDREKSON, ${ }^{1}$ VICTOR TORRES-COMPANY ${ }^{1}$ \\ ${ }^{1}$ Department of Microtechnology and Nanoscience(MC2), Photonics Laboratory, Chalmers University of Technology, SE-41296 Göteborg, \\ Sweden \\ *Corresponding author: zhichao@chalmers.se
}

Received XX Month XXXX; revised XX Month, XXXX; accepted XX Month XXXX; posted XX Month XXXX (Doc. ID XXXXX); published XX Month XXXX

Silicon nitride is a dielectric material widely used for applications in linear and nonlinear optics. It has an ultrabroad transparency window, low intrinsic loss and a refractive index that allows for moderate optical field confinement in waveguides. The chemical composition of this material can be precisely set during the fabrication process, leading to an extra degree of freedom for tailoring the optical and mechanical properties of photonic chips. Silicon-rich silicon nitride waveguides are appealing for nonlinear optics because they have higher nonlinear Kerr coefficient and refractive index than what is possible with stoichiometric silicon nitride. This is a direct consequence of the increased silicon content. However, silicon-rich silicon nitride waveguides typically display higher absorption losses. In this Letter, we report low loss $(\sim 0.4 \mathrm{~dB} / \mathrm{cm})$ silicon-rich silicon nitride waveguides. The structures feature high optical confinement and can be engineered with low anomalous dispersion. We find an optimum silicon composition that overcomes optical losses associated to $\mathrm{N}-\mathrm{H}$ bonds in the telecom band through an annealing process. Based on this technology, we successfully fabricate microresonators with mean quality factors $(Q) \sim 0.8 \times 106$ in the $C$ and $L$ bands. Broadband coherent microresonator frequency combs are generated in this platform, indicating its potential for efficient Kerr nonlinear optics.

http://dx.doi.org/10.1364/OL.99.099999

Stoichiometric silicon nitride $\left(\mathrm{Si}_{3} \mathrm{~N}_{4}\right)$ is a widely used material for passive integrated optics due to its complementary metal-oxide semiconductor compatibility, ultra-broad transparency window and low optical loss [1]. This platform has emerged as analternative to silicon for nonlinear applications in the near infrared due to the absence of nonlinear losses [2]. Silicon nitride has facilitated applications in self-referencing of low-power mode-locked lasers [3], octave-spanning microresonators combs via dispersive wave engineering [4,5] and wavelength conversion in microresonators at the single-photon level [6].

These applications require precise phase matching, which is attained by carefully engineering the cross-section geometry of the waveguide. For a silicon nitride core surrounded by silica cladding, the waveguide needs to be quite thick (typically above $700 \mathrm{~nm}$ ). Fabricating such thick films with high yield is challenging owing to the large tensile stress in as-deposited films, which can result in the formation of cracks crossing the photonic devices [7]. Notwithstanding, there has been dramatic progress in overcoming the challenges associated with fabricating thick silicon nitride waveguides. These include e.g. avoiding high temperature annealing in low-pressure chemical vapor deposition (LPCVD) [8] or using deuterium as precursor gas in a plasma-enhanced deposition (PECVD) process [9]. However, devices with the highest performance are achieved via LPCVD using dichlorosilane (DCS) and ammonia $\left(\mathrm{NH}_{3}\right)$ as precursor gases in combination with high temperature annealing [10-12]. Here, the challenges associated with the formation of cracks can be overcome by crack barrier trenches [11,13] and in an especially pre-patterned silica preform [14]. The latter approach has led to highly reproducible ultra-high Q microresonators approaching the fundamental intrinsic losses in stoichiometric silicon nitride films [12].

An alternative manner to overcome the high tensile stress in silicon nitride is varying the composition of the material itself. In particular, an increase of silicon content with respect to $\mathrm{Si}_{3} \mathrm{~N}_{4}$ could dramatically reduce the film stress [15]. The resulting material is known as silicon-rich silicon nitride (SiRN). It has been widely used for the fabrication of microelectromechanical systems and linear optics applications. Moreover, this material holds enormous potential for nonlinear optics as well. It has recently been discovered that increasing the silicon content yields both higher Kerr nonlinear coefficient [16-18] and, interestingly, higher secondorder nonlinear susceptibility [19]. Recent demonstrations using this emerging material platform include octave-spanning supercontinuum generation [20], parametric amplification [21] and broadband wavelength conversion [22]. 
One key drawback with SiRN is its increased linear absorption losses due to the formation of silicon nanocrystals [23]. This problem is accentuated by the extra difficulty in getting rid of $\mathrm{N}-\mathrm{H}$ bonds introduced during film deposition because using high temperature annealing forms silicon clusters that originate from out-diffusion from the substrate [24]. Some efforts have been made to get rid of $\mathrm{N}-\mathrm{H}$ bonds [25], but strong absorption peaks at the telecom window remain. As a result, high confinement low-loss SiRN waveguides have not been demonstrated to date. This precludes many practical applications of this promising platform. In this letter, we overcome these challenges by finding an optimal composition of SiRN that simultaneously provides high confinement, low stress and low loss $(\sim 0.4 \mathrm{~dB} / \mathrm{cm})$. We demonstrate high-Q microresonators and low-noise microresonator comb generation in this platform for the first time. Parts of this work were presented in [26]. Here we provide a more detailed study including ultrabroadband characterization of the films, dispersion measurements and low-noise frequency comb generation.

First, we seek the optimal quality and stoichiometry of SiRN films. We deposit our films via LPCVD. The concentration of silicon was indirectly controlled by the ratio between the precursor gases DCS and $\mathrm{NH}_{3}$ [16], and the SiRN film was deposited on 3" Si wafer with 3 $\mu \mathrm{m}$ thermally oxidized $\mathrm{SiO}_{2}$. Our previous results using this approach indicate absence of two-photon absorption [22] and a highly reproducible control of the refractive index [16]. Restricting the gas ratio between 3.24 and 7.15 is critical because the silicon content hardly increases after annealing at $1100{ }^{\circ} \mathrm{C}$ [24], thus indicating that there is little formation of Si clusters during this step. We found that a gas ratio of 3.9 is the minimum necessary to provide crack-free films with thickness $\sim 650 \mathrm{~nm}$, i.e. enough to simultaneously enable waveguide design with high optical confinement and anomalous dispersion. Such ratio is at least 4 times larger than the typically required ratio for $\mathrm{Si}_{3} \mathrm{~N}_{4}$, butbelow the value used in [17]. The nonlinear Kerr coefficient of this material composition is roughly twice higher than $\mathrm{Si}_{3} \mathrm{~N}_{4}$ [16]. In order to precisely measure the linear refractive index, a film with thickness $\sim 300 \mathrm{~nm}$ was deposited on a 3" Si wafer followed by annealing process at $1100^{\circ} \mathrm{C}$ under a nitrogen atmosphere for 3 hours. The complex refractive index of the samples was measured from $0.3 \mu \mathrm{m}$ to $30 \mu \mathrm{m}$ via ellipsometry [27], see Fig. 1(a). The real component of the refractive indexis 2.07 at $1550 \mathrm{~nm}$, slightly higher than the value for stoichiometric silicon nitride. The imaginary part of the refractive index indicates that the deposited SiRN film has an ultrabroad transparency window. We compare the results against stoichiometric silicon nitride films (gas ratio 0.3). In the longwavelength infrared, we note SiRN has lower absorption in the spectrum range from $7.3 \mu \mathrm{m}$ to $10.5 \mu \mathrm{m}$. Unlike with deuterated silicon nitride, we observe no absorption peaks in the relevant window around $2 \mu \mathrm{m}$. The slight difference of refractive index translates into a different bulk dispersion, as can be appreciated in Fig. 1(b). The simulated dispersion for the waveguide with height $670 \mathrm{~nm}$ and width $1900 \mathrm{~nm}$ indicates that small and flat anomalous dispersion could be simultaneously achieved in the $\mathrm{C}$ band. We note that the waveguide thickness required to achieve this result is smaller than with stoichiometric silicon nitride. This is a direct result of the higher refractive index in this platform and corresponding high optical field confinement. The dispersion for the devices included in the following sections are shown in Fig. 1(b).
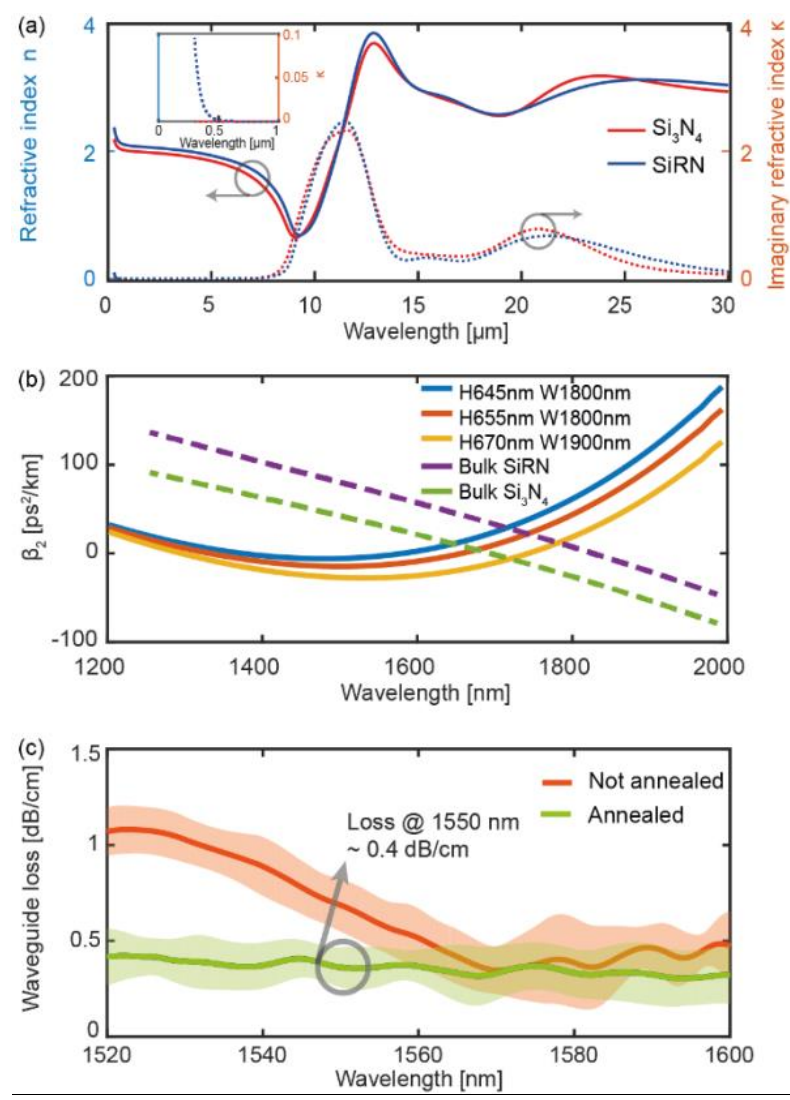

Fig. 1. (a) Complex refractive index comparison between our optimized $\mathrm{SiRN}$ and $\mathrm{Si}_{3} \mathrm{~N} 4$. The solid curves are the real part of the refractive index, and the dashed curves the imaginary (loss) component. (b) Simulated dispersion of SiRN waveguides for different cross-section geometrics (height, H, and width, W), SiRN bulk material and Si3N4. (c) Measured propagation loss for TE-polarization of straight waveguides with height $645 \mathrm{~nm}$ and width $1800 \mathrm{~nm}$. Colored lines show mean values and the shadowed areas show the standard deviation measured over 5 different waveguides.

After achieving high quality SiRN films, we fabricated straight waveguides in order to get a deeper understanding of this platform. A film with thickness $\sim 645 \mathrm{~nm}$ was deposited on a $3 \mu \mathrm{m}$ thermally oxidized 3" Si wafer. We used a similar process flow as in [17]. The exposure time of deep ultra-violet (DUV) lithography was optimized to achieve little shrinkage of waveguide width compared to the mask design. The SiRN film was dry etched using $\mathrm{CHF}_{3}$ and $\mathrm{O}_{2}$ gases. Standard cleaning followed by annealing process at $1100^{\circ} \mathrm{C}$ under a nitrogen atmosphere for 3 hours was applied after dry etching. Finally, the waveguides were cladded with $3 \mu \mathrm{m}$ PECVD $\mathrm{SiO}_{2}$. Importantly, no cracks appeared since we fabricated waveguides immediately after film deposition. However, we noticed that some cracks could appear at the edge of the wafers for unpatterned films after a few weeks. A thermal cycling process [7], which simultaneously reduces film stress and facilitates the outgas of hydrogen impurities [27], could be introduced if long storage times or thicker films were required. We separately measured the propagation losses of straight waveguides with and without annealing process. The loss of the fabricated waveguides was characterized by the cut-back method using three different lengths $(1.46,3.60,5.06 \mathrm{~cm})$. Fig. 1 (c) shows the mean value of propagation 
loss for the fundamental TE mode and its standard deviation over 5 waveguides on the same wafer. The absorption peak at $1520 \mathrm{~nm}$ that originates from residual $\mathrm{N}-\mathrm{H}$ bonds within the unannealed waveguides is clearly visible. This absorption peak was successfully eliminated by the annealing process without incurring extra loss. The average propagation loss of the annealed waveguides is $0.4 \pm$ $0.1 \mathrm{~dB} / \mathrm{cm}$ at $1550 \mathrm{~nm}$, three times lower than the value reported in [16] for the same composition.

We also fabricated microring resonators based on this platform. For the fabrication of microring resonators, we resorted to electron beam lithography (EBL) instead of DUV lithography, since our DUV lithography recipe could not attain the narrow gaps $\sim 400 \mathrm{~nm}$ that are needed to achieve critical coupling for the ring radii of $100 \mu \mathrm{m}$. Electron beam resist Ma-N 2405 was used as mask layer to pattern the microring resonators. Similar fabrication flow as described above for straight waveguides was used to fabricate the microring resonators. $\mathrm{SiO}_{2}$ with thickness $\sim 500 \mathrm{~nm}$ was deposited using LPCVD under $800{ }^{\circ} \mathrm{C}$ prior to PECVD $\mathrm{SiO}_{2}$ deposition since $\mathrm{SiO}_{2}$ deposited by LPCVD under high temperature has much better gap filling ability compared with PECVD $\mathrm{SiO}_{2}$. We fabricated rings with radii of $100 \mu \mathrm{m}$, corresponding to $220 \mathrm{GHz}$ free spectral range (FSR). The width and height of the ring waveguides are $1800 \mathrm{~nm}$ and $655 \mathrm{~nm}$. We used a similar method to [28] to measure the dispersion and quality factor of our devices. The measured integrated dispersion $\left(D_{\text {int }}=\omega_{\mu}-\omega_{0}-\mu D_{1}=D_{2} \mu_{2} / 2+D_{3} \mu_{3} / 6+\ldots\right.$ where $\omega_{\mu}$ is the angular frequency of the $\mu$-th resonance relative to the reference resonance $\omega_{0}$ and $D_{1}$ is the FSR [29]) and $Q_{i}$ factors over the range from 1520 to $1620 \mathrm{~nm}$ are shown in Fig. 2(a) and Fig. 2(b). The measured $D_{2} / 2 \pi$ is $300 \mathrm{kHz}$, corresponding to a group velocity dispersion coefficient of $-7 \mathrm{ps} 2 / \mathrm{km}\left(\beta_{2}=-\mathrm{n}_{0} \mathrm{D}_{2} / \mathrm{cD}_{12}\right)$ at $1560 \mathrm{~nm}$, close to our simulation result $(-12 \mathrm{ps} 2 / \mathrm{km})$. The mean $\mathrm{Q}_{\mathrm{i}}$ over the measurement bandwidth is $\sim 0.8 \times 10$ (equivalent to $\sim$ $0.45 \mathrm{~dB} / \mathrm{cm}$ ). A representative resonance at $1590 \mathrm{~nm}$ with $\mathrm{Q}_{\mathrm{i}} \sim 1 \mathrm{x}$ 106 is given in the inset of Fig. 2(a). The lowest $Q_{i}$ at $1558 \mathrm{~nm}$ is due to an avoided mode crossing, as can be observed in the dispersion profile in Fig. 2(b). We measured the line edge roughness of our waveguides based on scanning electron microscope images. The obtained root mean square of roughness and correlation length were $\left(\sigma, L_{c}\right)=(3.6 \mathrm{~nm}, 40 \mathrm{~nm})$ which are close to what we measured in [17]. This analysis indicates that scattering losses are still the main dominating contribution in our devices. Further optimized dry etching recipe [10] for SiRN could provide better losses. Moreover, the $\mathrm{Q}_{\mathrm{i}}$ around $1530 \mathrm{~nm}$ is still slightly lower than that around $1600 \mathrm{~nm}$, indicating that there are still some losses due to residual $\mathrm{N}-\mathrm{H}$ bonds. These could be further improved by adapting a thermal cycling process in our film deposition [7].

Next, we investigate microresonator frequency comb generation in our devices. The threshold power (coupled in the bus waveguide) for parametric oscillation in a $220 \mathrm{GHz}$ FSR microresonator was $21 \mathrm{~mW}$. We then increased on-chip pump power to $\sim 150 \mathrm{~mW}$ to seek for broadband microresonator combs. We tuned the laser frequency with a speed $\sim 10 \mathrm{~nm} / \mathrm{s}$ and recorded both the throughput and converted light power in the range $1450-1500 \mathrm{~nm}$. We occasionally see soliton steps [29] in both throughput and comb power when scanning the resonance around $1539 \mathrm{~nm}$. The evolution of frequency combs while the pump laser was swept from the blue to the red side of the resonance was measured by an optical spectrum analyzer (YOKOGAWA AQ6375B), and the results are
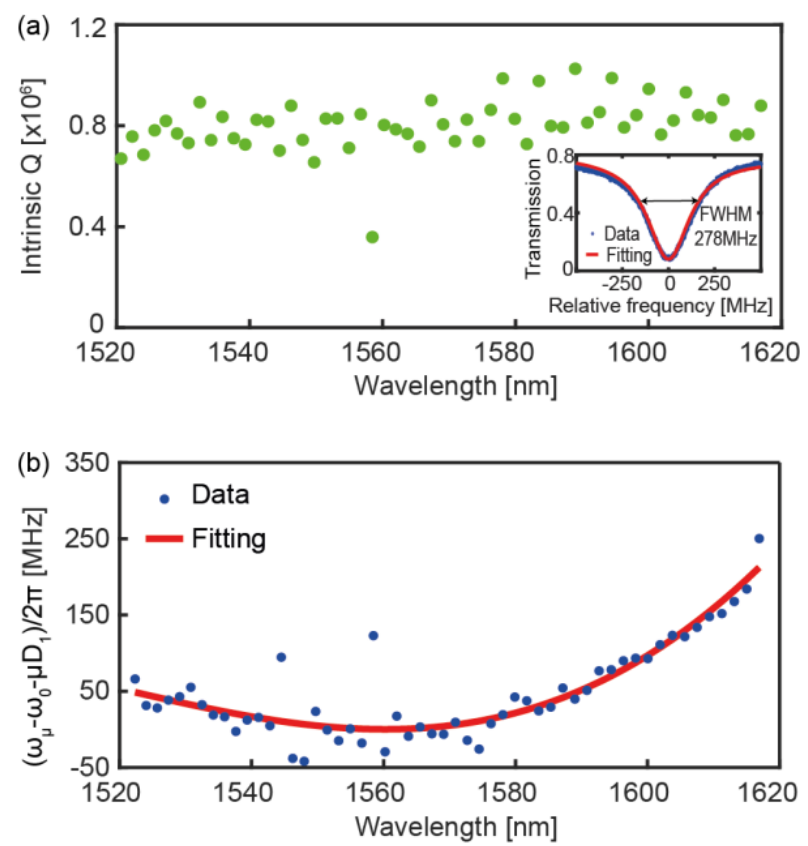

Fig. 2. (a) Intrinsic $Q$ of each resonance from the fundamental TE mode family. Inset: measurement and fitting result of the resonance. (b) Measured integrated dispersion of the fundamental TE mode family.

shown in Fig.3 (a). The corresponding radio-frequency (RF) spectra measured by an electrical spectrum analyzer (ESA) are shown in Fig. 3(b). One can clearly observe a transition from a coherent modulation instability region (I) to an incoherent state (II). The chaotic comb state could occasionally transit into a low noise state, which is shown in Fig. 3(a)(III). Its optical spectrum combined with low noise RF spectrum seems to indicate a solitonic crystal state [30].

We also fabricated microring resonators with radii of $22 \mu \mathrm{m}$, corresponding to $1 \mathrm{THz}$ FSR. The width and height of the ring waveguides were $1300 \mathrm{~nm}$ and $655 \mathrm{~nm}$. This design ensures low anomalous dispersion (-8 ps2/ $\mathrm{km}$ at $1542 \mathrm{~nm})$. The loaded Q is 280000, and on-chip power $\sim 24 \mathrm{~mW}$ is needed for parametric oscillation. An on-chip power $\sim 100 \mathrm{~mW}$ was used to pump this device at $1542 \mathrm{~nm}$. Further analysis of the parametric threshold on multiple devices leads us to estimate $\mathrm{n}_{2} \sim 5.0 \pm 1.6 \times 10-19 \mathrm{~m} 2 / \mathrm{W}$, which is twice higher than the corresponding value for $\mathrm{Si}_{3} \mathrm{~N}_{4}$ [31]. Similar frequency comb evolution as with the $220 \mathrm{GHz}$ FSR device was observed during wavelength tuning of the pump laser i.e., starting from a coherent MI state, transitioning to chaotic state and occasionally reaching a low noise state. Here, a broadband frequency comb from $1300 \mathrm{~nm}$ to $2000 \mathrm{~nm}$ was successfully achieved, as indicated by the optical and RF spectra in Figs. 3(c) and (d). We have not been able to observe single solitons. We believe that this is due to practical difficulties in stopping the laser at the desired wavelength while fast tuning. This could be solved in the future by e.g. rapid tuning with a single-sideband modulator [32] or realizing thermo-optic tuning of the cavity resonances [33].

In conclusion, we found an optimal ratio of precursor gases DCS and $\mathrm{NH}_{3}$ to deposit thick SiRN films which simultaneously enable crack-free formation and low optical losses. Low loss waveguides and high Q microring resonators have been achieved based on this 

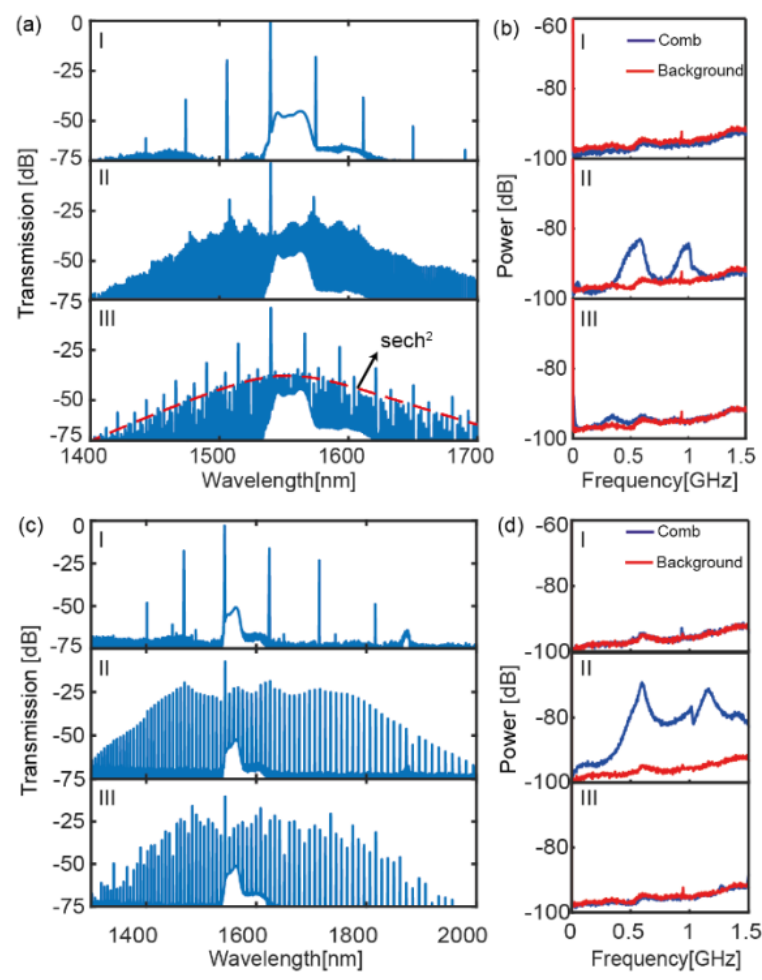

Fig. 3 (a) Optical spectrum of stable MI comb (I), chaotic MI comb (II) and its transition to low noise comb (III) based on microring resonator with $220 \mathrm{GHz}$ FSR. (b) RF spectra corresponding to comb states in (a). (c) and (d) are similar to (a) and (b) but based on microring resonator with 1 THz FSR.

emerging platform. Broadband low-noise microresonator combs have been achieved in microrings with both $1 \mathrm{THz}$ and $220 \mathrm{GHz}$ FSRs. The low loss, large nonlinearity and simplicity of depositing thick films make this a promising platform for Kerr nonlinear optics. The raw data that accompanies the figures in this paper, including the recipes used for fabricating devices, is accessible in https://doi.org/10.5281/zenodo.2535437.

Funding. This project is funded by the Swedish Research Council (VR, 2015-00535, 2016-03960 and 2016-06077) and the European Research Council (ERC, 771410).

Acknowledgment. The authors thank Clemens J. Krückel for developing the initial fabrication flows and building the measurementenvironment, and the cleanroom staff from Chalmers nanofabrication laboratory for fruitful discussions.

\section{References}

1. A. Rahim, E. Ryckeboer, A. Z. Subramanian, S. Clemmen, B. Kuyken, A. Dhakal, A. Raza, A. Hermans, M. Muneeb, S. Dhoore, Y. Li, U. Dave, P. Bienstman, N. Le Thomas, G. Roelkens, D. Van Thourhout, P. Helin, S. Severi, X. Rottenberg, and R. Baets, J. Light. Technol. 35, 639-649 (2017).

2. L. Razzari, D. Duchesne, M. Ferrera, R. Morandotti, S. Chu, B. E. Little, and D. J. Moss, Nat. Photonics 4, 41-45 (2010).

3. A. Klenner, A. S. Mayer, A. R. Johnson, K. Luke, M. R. E. Lamont, Y. Okawachi, M. Lipson, A. L. Gaeta, and U. Keller, Opt. Express 24, 11043-11053 (2016).

4. Q. Li, T. C. Briles, D. A. Westly, T. E. Drake, J. R. Stone, B. R. llic, S. A. Diddams, S. B. Papp, and K. Srinivasan, Optica 4,193-203 (2016).
5. M. H. P. Pfeiffer, C. Herkommer, J. Liu, H. Guo, M. Karpov, E. Lucas, M. Zervas, and T. J. Kippenberg, Optica 4, 684-691 (2017).

6. Q. Li, M. Davanço, and K. Srinivasan, Nat. Photonics 10, 406-414 (2016).

7. A. Gondarenko, J. S. Levy, and M. Lipson, Opt. Express 17, 11366-11370 (2009).

8. H. El Dirani, A. Kamel, M. Casale, S. Kerdiles, C. Monat, X. Letartre, M. Pu, L. K. Oxenløwe, K. Yvind, and C. Sciancalepore, Appl. Phys. Lett. 113, 081102 (2018).

9. J. Chiles, N. Nader, D. D. Hickstein, S. P. Yu, T. C. Briles, D. Carlson, H. Jung, J. M. Shainline, S. Diddams, S. B. Papp, S. W. Nam, and R. P. Mirin, Opt. Lett. 43, 7-10 (2018).

10. X. Ji, F. A. S. Barbosa, S. P. Roberts, A. Dutt, J. Cardenas, Y. Okawachi, A. Bryant, A. L. Gaeta, and M. Lipson, Optica 4, 619-624 (2017).

11. Y. Xuan, Y. Liu, L. T. Varghese, A. J. Metcalf, X. Xue, P.-H. Wang, K. Han, J. A. Jaramillo-Villegas, A. Al Noman, C. Wang, S. Kim, M. Teng, Y. J. Lee, B. Niu, L. Fan, J. Wang, D. E. Leaird, A. M. Weiner, and M. Qi, Optica 3, 1171-1180 (2016).

12. J. Liu, A. S. Raja, M. Karpov, B. Ghadiani, H. P. Martin, N. J. Engelsen, H. Guo, M. Zervas, and T. J. Kippenberg, Optica 5, 29-31 (2018).

13. K. Luke, A. Dutt, C. B. Poitras, and M. Lipson, Opt. Express 21, 22829-22833 (2013).

14. M. H. P. Pfeiffer, A. Kordts, V. Brasch, M. Zervas, M. Geiselmann, J. D. Jost, and T. J. Kippenberg, Optica 3, 20-25 (2016).

15. M. Cheng, W. Ho, C. P. Chang, W. S. Huang, and R.-S. S. Huang, Device Process Technol. MEMS Microelectron. 3892, 152-157 (1999).

16. C. J. Krückel, A. Fülöp, Z. Ye, P. A. Andrekson, and V. Torres-Company, Opt. Express 25, 770-776 (2017).

17. C. J. Krückel, A. Fülöp, T. Klintberg, J. Bengtsson, P. A. Andrekson, and V. Torres-Company, Opt. Express 23, 25827-25837 (2015).

18. C. Lacava, S. Stankovic, A. Z. Khokhar, T. D. Bucio, F. Y. Gardes, G. T. Reed, D. J. Richardson, and P. Petropoulos, Sci. Rep. 7, 22 (2017).

19. K. Koskinen, R. Czaplicki, A. Slablab, T. Ning, A. Hermans, B. Kuyken, V. Mittal, G. S. Murugan, T. Niemi, R. Baets, and M. Kauranen, Opt. Lett. 42, 5030-5033 (2017)

20. X. Liu, M. Pu, B. Zhou, C. J. Krückel, A. Fülöp, V. Torres-Company, and M. Bache, Opt. Lett. 41, 2719-2722 (2016).

21. K. J. A. Ooi, D. K. T. Ng, T. Wang, A. K. L. Chee, S. K. Ng, Q. Wang, L. K. Ang, A. M. Agarwal, L. C. Kimerling, and D. T. H. Tan, Nat. Commun. 8, 13878 (2017).

22. M. R. Dizaji, C. J. Krückel, A. Fülöp, P. A. Andrekson, V. Torres-Company, and L. R. Chen, Opt. Express 25, 12100-12108 (2017).

23. V. E. Vamvakas, N. Vourdas, and S. Gardelis, Microelectron. Reliab. 47, 794-797 (2007).

24. K. N. Andersen, W. E. Svendsen, T. Stimpel-Lindner, T. Sulima, and H. Baumgärtner, Appl. Surf. Sci. 243, 401-408 (2005).

25. H. Mertens, K. N. Andersen, and W. E. Svendsen, IEEE Opt. Commun. Eur. Conf. P1, 38 (2002).

26. Z. Ye, A. Fülöp, Ó. B. Helgason, P. A. Andrekson, and V. Torres-company, in Conference on Lasers and Electro-Optics, OSA Technical Digest (online) (Optical Society of America, 2018), paper STu3F.1.

27. K. Luke, Y. Okawachi, M. R. E. Lamont, A. L. Gaeta, and M. Lipson, Opt. Lett. 40, 4823-4826 (2015).

28. S. W. Huang, H. Zhou, J. Yang, J. F. McMillan, A. Matsko, M. Yu, D. L. Kwong, L. Maleki, and C. W. Wong, Phys. Rev. Lett. 114, 053901 (2015).

29. T. Herr, V. Brasch, J. D. Jost, C. Y. Wang, N. M. Kondratiev, M. L. Gorodetsky, and T. J. Kippenberg, Nat. Photonics 8, 145-152 (2012).

30. D. C. Cole, E. S. Lamb, P. Del'Haye, S. A. Diddams, and S. B. Papp, Nat. Photonics 11, 671-676 (2017).

31. K. Ikeda, R. E. Saperstein, N. Alic, and Y. Fainman, Opt. Express 16, 1298712994 (2008).

32. J. R. Stone, T. C. Briles, T. E. Drake, D. T. Spencer, D. R. Carlson, S. A. Diddams, and S. B. Papp, Phys. Rev. Lett. 121, 63902 (2018).

33. C. Joshi, J. K. Jang, K. Luke, X. Ji, S. A. Miller, A. Klenner, Y. Okawachi, M. Lipson, and A. L. Gaeta, Opt. Lett. 41, 2565-2568 (2016). 


\section{Last page:}

1. A. Rahim, E. Ryckeboer, A. Z. Subramanian, S. Clemmen, B. Kuyken, A. Dhakal, A. Raza, A. Hermans, M. Muneeb, S. Dhoore, Y. Li, U. Dave, P. Bienstman, N. Le Thomas, G. Roelkens, D. Van Thourhout, P. Helin, S. Severi, X. Rottenberg, and R. Baets, "Expanding the Silicon Photonics Portfolio with Silicon Nitride Photonic Integrated Circuits," J. Light. Technol. 35, 639-649 (2017).

2. L. Razzari, D. Duchesne, M. Ferrera, R. Morandotti, S. Chu, B. E. Little, and D. J. Moss, "CMOS-compatible integrated optical hyperparametric oscillator," Nat. Photonics 4, 41-45 (2010).

3. A. Klenner, A. S. Mayer, A. R. Johnson, K. Luke, M. R. E. Lamont, Y. Okawachi, M. Lipson, A. L. Gaeta, and U. Keller, "Gigahertz frequency comb offset stabilization based on supercontinuum generation in silicon nitride waveguides," Opt. Express 24, 1104311053 (2016)

4. Q. Li, T. C. Briles, D. A. Westly, T. E. Drake, J. R. Stone, B. R. Ilic, S. A. Diddams, S. B. Papp, and K. Srinivasan, "Stably accessing octavespanning microresonator frequency combs in the soliton regime," 4, (2016).

5. M. H. P. Pfeiffer, C. Herkommer, J. Liu, H. Guo, M. Karpov, E. Lucas, M. Zervas, and T. J. Kippenberg, "Octave-spanning dissipative Kerr soliton frequency combs in $\mathrm{Si}_{3} \mathrm{~N}_{4}$ microresonators," Optica 4, 684 691 (2017).

6. Q. Li, M. Davanço, and K. Srinivasan, "Efficient and low-noise single-photon-level frequency conversion interfaces using silicon nanophotonics," Nat. Photonics 10, 406-414 (2016).

7. A. Gondarenko, J. S. Levy, and M. Lipson, "High confinement micron-scale silicon nitride high $\mathrm{Q}$ ring resonator," Opt. Express 17, 11366-11370 (2009).

8. H. El Dirani, A. Kamel, M. Casale, S. Kerdiles, C. Monat, X. Letartre, M. Pu, L. K. Oxenløwe, K. Yvind, and C. Sciancalepore, "Annealingfree $\mathrm{Si}_{3} \mathrm{~N}_{4}$ frequency combs for monolithic integration with $\mathrm{Si}$ photonics," Appl. Phys. Lett. 113, 081102 (2018).

9. J. Chiles, N. Nader, D. D. Hickstein, S. P. Yu, T. C. Briles, D. Carlson, H. Jung, J. M. Shainline, S. Diddams, S. B. Papp, S. W. Nam, and R. P. Mirin, "Deuterated silicon nitride photonic devices for broadband optical frequency comb generation," Opt. Lett. 43, 710 (2018).

10. X. Ji, F. A. S. Barbosa, S. P. Roberts, A. Dutt, J. Cardenas, Y. Okawachi, A. Bryant, A. L. Gaeta, and M. Lipson, "Ultra-low-loss on-chip resonators with sub-milliwatt parametric oscillation threshold," Optica 4, 619-624 (2017).

11. Y. Xuan, Y. Liu, L. T. Varghese, A. J. Metcalf, X. Xue, P.-H. Wang, K. Han, J. A. Jaramillo-Villegas, A. Al Noman, C. Wang, S. Kim, M. Teng, Y. J. Lee, B. Niu, L. Fan, J. Wang, D. E. Leaird, A. M. Weiner, and M. Qi, "High-Q silicon nitride microresonators exhibiting lowpower frequency comb initiation," Optica 3, 1171-1180 (2016).

12. J. Liu, A. S. Raja, M. Karpov, B. Ghadiani, H. P. Martin, N. J. Engelsen, H. Guo, M. Zervas, and T. J. Kippenberg, "Ultralowpower chip-based soliton microcombs for photonic integration," Optica 5, 29-31 (2018).

13. K. Luke, A. Dutt, C. B. Poitras, and M. Lipson, "Overcoming $\mathrm{Si}_{3} \mathrm{~N}_{4}$ film stress limitations for high quality factor ring resonators," Opt. Express 21, 22829-22833 (2013).

14. M. H. P. Pfeiffer, A. Kordts, V. Brasch, M. Zervas, M. Geiselmann, J. D. Jost, and T. J. Kippenberg, "Photonic Damascene Process for Integrated High-Q Microresonator Based Nonlinear Photonics," Optica 3, 20-25 (2016).

15. M. Cheng, W. Ho, C. P. Chang, W. S. Huang, and R.-S. S. Huang, "Ultralow-stress silicon-rich nitride films for microstructure fabrication," Device Process Technol. MEMS Microelectron. 3892, 152-157 (1999).

16. C. J. Krückel, A. Fülöp, Z. Ye, P. A. Andrekson, and V. TorresCompany, "Optical bandgap engineering in nonlinear silicon nitride waveguides," Opt. Express 25, 770-776 (2017).

17. C. J. Krückel, A. Fülöp, T. Klintberg, J. Bengtsson, P. A. Andrekson, and $\mathrm{V}$. Torres-Company, "Linear and nonlinear characterization of low-stress high-confinement silicon-rich nitride waveguides," Opt. Express 23, 25827-25837 (2015).

18. C. Lacava, S. Stankovic, A. Z. Khokhar, T. D. Bucio, F. Y. Gardes, G. T. Reed, D. J. Richardson, and P. Petropoulos, "Si-rich Silicon Nitride for Nonlinear Signal Processing Applications," Sci. Rep. 7, 22 (2017).

19. K. Koskinen, R. Czaplicki, A. Slablab, T. Ning, A. Hermans, B. Kuyken, V. Mittal, G. S. Murugan, T. Niemi, R. Baets, and M. Kauranen, "Enhancement of bulk second-harmonic generation from silicon nitride films by material composition," Opt. Lett. $\mathbf{4 2}$, 5030-5033 (2017).

20. X. Liu, M. Pu, B. Zhou, C. J. Krückel, A. Fülöp, V. Torres-Company, and $\mathrm{M}$. Bache, "Octave-spanning supercontinuum generation in a silicon-rich nitride waveguide," Opt. Lett. 41, 2719-2722 (2016).

21. K. J. A. Ooi, D. K. T. Ng, T. Wang, A. K. L. Chee, S. K. Ng, Q. Wang, L. K. Ang, A. M. Agarwal, L. C. Kimerling, and D. T. H. Tan, "Pushing the limits of CMOS optical parametric amplifiers with USRN:Si ${ }_{7} \mathrm{~N}_{3}$ above the two-photon absorption edge," Nat. Commun. 8, 13878 (2017).

22. M. R. Dizaji, C. J. Krückel, A. Fülöp, P. A. Andrekson, V. TorresCompany, and L. R. Chen, "Silicon-rich nitride waveguides for ultra-broadband nonlinear signal processing," Opt. Express 25, 12100-12108 (2017).

23. V. E. Vamvakas, N. Vourdas, and S. Gardelis, "Optical characterization of Si-rich silicon nitride films prepared by low pressure chemical vapor deposition," Microelectron. Reliab. 47, 794-797 (2007).

24. K. N. Andersen, W. E. Svendsen, T. Stimpel-Lindner, T. Sulima, and H. Baumgärtner, "Annealing and deposition effects of the chemical composition of silicon-rich nitride," Appl. Surf. Sci. 243, 401-408 (2005).

25. H. Mertens, K. N. Andersen, and W. E. Svendsen, "Optical Loss Analysis of Silicon Rich Nitride Waveguides," IEEE Opt. Commun. Eur. Conf. P1, 38 (2002).

26. Z. Ye, A. Fülöp, Ó. B. Helgason, P. A. Andrekson, and V. Torrescompany, "Low Loss Silicon-Rich Silicon Nitride for Nonlinear Optics," in Conference on Lasers and Electro-Optics, OSA Technical Digest (online) (Optical Society of America, 2018), paper STu3F.1.

27. K. Luke, Y. Okawachi, M. R. E. Lamont, A. L. Gaeta, and M. Lipson, "Broadband mid-infrared frequency comb generation in a $\mathrm{Si}_{3} \mathrm{~N}_{4}$ microresonator," Opt. Lett. 40, 4823-4826 (2015).

28. S. W. Huang, H. Zhou, J. Yang, J. F. McMillan, A. Matsko, M. Yu, D. L. Kwong, L. Maleki, and C. W. Wong, "Mode-locked ultrashort pulse generation from on-chip normal dispersion microresonators," Phys. Rev. Lett. 114, 053901 (2015).

29. T. Herr, V. Brasch, J. D. Jost, C. Y. Wang, N. M. Kondratiev, M. L. Gorodetsky, and T. J. Kippenberg, "Temporal solitons in optical microresonators," Nat. Photonics 8, 145-152 (2012).

30. D. C. Cole, E. S. Lamb, P. Del'Haye, S. A. Diddams, and S. B. Papp, "Soliton crystals in Kerr resonators," Nat. Photonics 11, 671-676 (2017).

31. K. Ikeda, R. E. Saperstein, N. Alic, and Y. Fainman, "Thermal and Kerr nonlinear properties of plasma-deposited silicon nitride/silicon dioxide waveguides," Opt. Express 16, 12987-12994 (2008)

32. J. R. Stone, T. C. Briles, T. E. Drake, D. T. Spencer, D. R. Carlson, S A. Diddams, and S. B. Papp, "Thermal and Nonlinear DissipativeSoliton Dynamics in Kerr-Microresonator Frequency Combs," Phys. Rev. Lett. 121, 63902 (2018).

33. C. Joshi, J. K. Jang, K. Luke, X. Ji, S. A. Miller, A. Klenner, Y. Okawachi, M. Lipson, and A. L. Gaeta, "Thermally Controlled Comb Generation and Soliton Modelocking in Microresonators," Opt. Lett.41, 2565-2568 (2016). 